\title{
Reconhecimento de expressões faciais e cenas de valência emocional apresentadas em alta restrição temporal ${ }^{1}$
}

\author{
Rui de Moraes Júnior \\ Universidade de São Paulo - Ribeirão Preto \\ Université de Montréal \\ Beatriz Francisco Barbosa \\ Fernanda Pires Garcia \\ Flavia Helen Moreira da Silva \\ Julia Ribeiro \\ Márcia Viana Amaral \\ Sérgio Sheiji Fukusima \\ Universidade de São Paulo - Ribeirão Preto
}

\begin{abstract}
Resumo
Este estudo investigou o reconhecimento de flashes de imagens de conteúdo afetivo de diferentes modalidades (faces e cenas) e valências emocionais (agradáveis, desagradáveis e neutras). Os resultados evidenciaram que a diferença de saliência perceptual entre os estímulos pode se sobrepor aos efeitos da valência emocional. O reconhecimento de cenas e de faces agradáveis não se diferenciou; o mesmo aconteceu para cenas e faces desagradáveis. Por outro lado, as valências apresentaram diferenças: imagens agradáveis foram reconhecidas mais rapidamente em relação às desagradáveis e faces agradáveis foram mais reconhecidas que as neutras. Suspeita-se, conforme literatura existente, que imagens desagradáveis apresentadas rapidamente enfraqueçam a ativação da amígdala e intensifiquem a ativação do córtex pré-frontal, que realiza um processamento de informações utilizando um tempo maior.
\end{abstract}

Palavras-chave: percepção subliminar; emoção; valência emocional; IAPS; POFA.

\begin{abstract}
Recognition of facial expressions and scenes of emotional valence presented at high temporal constraint. This study investigated the recognition of affective images flashes of different stimuli modalities (faces and scenes) and emotional valences (pleasant, unpleasant and neutral). Results showed that the difference among stimuli's perceptual salience can overlap the effects of emotional valence. As the recognition between pleasant scenes and faces, unpleasant ones did not differ. On the other hand the emotional valences showed differences: pleasant images were recognized faster compared to unpleasant images and pleasant faces were more recognized than neutral faces. Taking into account literature these results suggest that unpleasant images rapidly presented weaken activation in the amygdala and intensify it in the prefrontal cortex, which takes longer to process information.
\end{abstract}

Keywords: subliminal perception; emotion; emotional valence; IAPS; POFA.

\section{Resumen}

Reconocimiento de expresiones faciales y escenas de valencia emocional presentadas con alta restricción temporal. El objetivo de este estudio fue investigar el reconocimiento de flashes de imágenes con contenido afectivo de diferentes modalidades (rostros y escenas) y valencias emocionales (agradables, desagradables y neutras). Los resultados mostraron que la diferencia de saliencia perceptiva entre los estímulos se puedes obre poner a los efectos de la valencia emocional. No hubo diferencia entre el reconocimiento de escenas y de rostros agradables; lo mismo ocurrió para escenas y rostros desagradables. Por otra parte, las valencias presentaron diferencias: imágenes agradables fueron reconocidas más rápidamente en comparación a las desagradables, y caras agradables fueron más reconocidas que las neutras. Según la literatura existente, inferimos que imágenes desagradables presentadas rápidamente debilitan la activación de la amígdala e intensifican la activación del córtex pre-frontal, que realiza el procesamiento de información en un tiempo mayor.

Palabras clave: percepción subliminar; emoción; valencia emocional; IAPS; POFA. 
U ma das evidências mais simples de emoção referese à manifestação de reações psicomotoras, geralmente acompanhadas de alterações neurovegetativas em resposta a um estímulo ambiental (Brandão, 2004). Em adição, Damásio (2000) afirma que as emoções podem ser concebidas, de modo abrangente, como coleções específicas e consistentes de respostas cognitivas e fisiológicas acionadas por sistemas cerebrais que preparam o organismo para a ação e a interação social. As emoções são moduladas por estímulos sensoriais do ambiente, em especial os de carga afetiva, e são responsáveis por mudanças profundas no corpo. O conjunto dessas mudanças é possibilitado por substratos neurais específicos no cérebro, que proporcionam as experiências emocionais. Evolutivamente, esses substratos neurais se localizaram em uma posição intermediária entre os mecanismos básicos de sobrevivência (regulação do metabolismo, reflexos simples, biologia da dor e do prazer) e a emergência dos mecanismos responsáveis pelo raciocínio. Aos mecanismos básicos de sobrevivência associam-se as emoções, que fazem parte do instrumental de regulação da vida e desempenham um papel importante na relação dos organismos com o mundo externo. No entanto, são os mecanismos responsáveis pelo raciocínio que estabelecem os sentimentos, elaborados pela consciência (Damásio, 2000).

Para Staats (1996), esse aparato não teria se desenvolvido e se mantido se não tivesse significado adaptativo para o comportamento dos organismos. Assim, a resposta emocional foi mantida pela evolução através do tempo para grande variedade de organismos complexos. O livro A expressão das emoções no homem e nos animais, de Charles Darwin (1872/2000), constituiu uma das primeiras fontes de informação sobre as emoções dentro da perspectiva evolucionista. Foram descritas reações de medo, choro, dor, ansiedade, mau humor, surpresa, alegria, horror, raiva, dentre outras, em faces humanas e de outros animais, considerando suas origens evolucionárias em relação à sua utilidade biológica (Millenson, 1967/1975).

A partir das descobertas de Darwin, várias pesquisas foram realizadas a fim de investigar a relação entre a percepção/ indução de emoções e a as expressões faciais. Estas desempenham um papel fundamental na apreciação de nosso ambiente físico e social. Por isso, são cruciais no desenvolvimento e na regulação dos relacionamentos interpessoais, sendo que muitas vezes são vistas como sinais externos de emoções (Frank \& Stennett, 2001). Por isso, dificuldades na percepção ou na expressão de emoções faciais frequentemente comprometem a adaptação social do indivíduo. Estímulos emocionais mais genéricos, como cenas de conteúdo emocional, são importantes para a sobrevivência da espécie, por exemplo, na medida em que indicam algum perigo (Dixon, 1971; Ohman, Lundqvist, \& Esteves, 2001).

Dada a sua importância, estímulos emocionais podem acarretar um aumento da sensibilidade perceptual e um processamento visual intensificado (Anderson \& Phelps, 2001). Esta suposição foi investigada por estudos que utilizaram condições marginais de estimulação, quando os estímulos estão presentes em intensidades muito fracas ou durante tempos de exposição extremamente curtos, nas quais os estímulos aparentemente não provocam nenhuma resposta de detecção pelos indivíduos. Essas condições são chamadas de estimulação subliminar (Schiffman, 2001). Várias pesquisas dão apoio a esta ideia. Estudos por meio de ressonância magnética funcional mostraram que as apresentações subliminares de estímulos emocionais, tanto faces como cenas, produziram alta ativação na amígdala (Sabatini et al., 2009; Whalen et al., 1998); região localizada no interior do lobo temporal medial, associada ao processamento de emoções. Krosnick, Metz, Jussim e Lynn (1992) verificaram que imagens que foram precedidas por flashes de estímulos agradáveis (imagem de gatinhos e de um casal romântico, por exemplo) foram classificadas mais positivamente do que as mesmas fotografias precedidas por estímulos desagradáveis (imagem de cadáver e de um rosto zangado, por exemplo). Ohman et al. (2001) mostraram que faces que expressam perigo são detectadas mais rapidamente que aquelas neutras. Cenas emocionais também foram discriminadas mais rapidamente que cenas neutras (Cuthbert, Schupp, Bradley, Birbaumer, \& Lang, 2000). Entretanto, pouco se sabe a respeito da apresentação subliminar de figuras com valências emocionais agradável, desagradável e neutro, sobre seu reconhecimento posterior.

Em geral, estudos com apresentação subliminar têm investigado a emoção a partir de duas diferentes modalidades de estímulos visuais: expressões faciais de emoção ou cenas de conteúdo emocional. Para Hatfield, Cacioppo e Rapson (1992) e Wild, Erb e Bartels (2001), as expressões faciais são usadas com frequência como indutoras de emoção. De acordo com Britton, Taylor, Sudheimer e Liberzon (2006), as expressões faciais de emoção podem provocar um maior reconhecimento/ percepção de emoções e, por isso, são usadas como sondas de reconhecimento. Já as cenas seriam mais indutoras de emoções, considerando que elas podem propiciar maior experiência direta da emoção. Porém, os autores afirmaram que, tanto cenas emocionalmente evocativas como faces com expressões emocionais ativam um conjunto semelhante de regiões: amígdala, hipocampo, ínsula, giro cingulado anterior, córtex préfrontal medial e córtex pré-frontal ventromedial.

Um grande número de estudos sobre valência emocional utiliza imagens do International Affective Picture System (IAPS) (Lang, Bradley, \& Cuthbert, 2008), um dos conjuntos de imagens mais amplamente usados como estímulos para investigar a emoção no laboratório. O IAPS consiste em uma coleção de imagens estáticas que retratam cenas carregadas de emoção como, por exemplo: mutilações, cobras, insetos, cenas de ataques, acidentes, contaminação, doenças, perdas, poluição, filhotes de cachorro, bebês, cenas de paisagem, entre outros (Mikels et al., 2005). As figuras podem ser classificadas de acordo com os estados psicofisiológicos que produzem, com base nas categorizações gerais de prazer, alerta e dominância. $O$ banco de imagens do IAPS é um instrumento eficiente na indução de diferentes estados emocionais e permite elevado nível de controle dos parâmetros de exposição e mensuração em contextos experimentais. Por isso, tem sido amplamente utilizado em pesquisas na área da cognição, do afeto, do comportamento, das 
atitudes sociais, da psicofisiologia, entre outras, tanto para estudar pacientes com distúrbios neuropsiquiátricos quanto populações saudáveis (Lasaitis, Ribeiro, Freire, \& Amodeo, 2008).

Outro instrumento de avaliação/indução de estados emocionais com grande nível de controle experimental e amplamente utilizado em pesquisas é o Pictures of Facial Affect (POFA). Ele foi elaborado por Ekman e Friesen (1976) e consiste em fotografias de expressões faciais que foram amplamente utilizadas em estudos transculturais. Para categorizar as expressões faciais, Ekman e Friesen (1978) desenvolveram o Facial ActionCoding System, FACS (a atual versão se chama FACE, de Facial Expression, Awareness, Compassion, Emotions). Este sistema foi elaborado a partir de minuciosa análise de uma amostra de cinco mil expressões faciais de adultos, gravadas em vídeo. Os autores registraram os movimentos faciais feitos por cada sujeito, chegando a um protótipo morfológico das emoções básicas: alegria, tristeza, raiva, nojo, surpresa e medo. Com esses protótipos que indicam o movimento das unidades de ação (músculos isolados ou em combinação com outros que são envolvidos em cada emoção básica), os autores pretenderam realizar uma análise objetiva das expressões faciais (Galvão, 2001).

Para Bueno e Primi (2003) um dos problemas mais evidentes relacionado à percepção de emoções é o de sua mensuração. Sem um instrumento confiável para mensurá-la não é possível conhecer objetivamente suas características funcionais e estruturais na mente humana. Considerando que as fotografias de faces retiradas das séries de Ekman e as imagens do IAPS são frequentemente utilizadas em estudos sobre percepção de emoções, torna-se necessário o desenvolvimento de estudos comparativos entre estes instrumentos. Isso deve ser efetuado para se testar a confiabilidade e funcionalidade dos mesmos, e para investigar se nosso sistema visual percebe estímulos de conteúdo emocional de diferentes modalidades de maneira distinta. Além disso, pouco se sabe a respeito do efeito da apresentação subliminar sobre o reconhecimento posterior de imagens com diferentes valências emocionais (agradáveis e desagradáveis) de imagens do IAPS e do POFA. Isto justifica estudos que comparem estímulos de ambos os bancos de imagens quando apresentados em situações limítrofes ou inconscientes.

Portanto, a partir da revisão realizada, investigou-se no presente estudo a influência de estímulos visuais agradáveis, desagradáveis e neutros quando apresentados muito brevemente em uma tarefa de reconhecimento. Também foi objetivo desse estudo comparar duas modalidades de estímulos, faces e cenas, em dois bancos de imagens amplamente utilizados na literatura: o IAPS e o POFA. Para isso, foram analisadas as médias das respostas corretas $(\mathrm{RC})$ e os tempos para emissão de respostas corretas (TR).

\section{Método}

\section{Participantes}

Participaram deste estudo 32 estudantes universitários (16 mulheres), com idade entre 18 e 23 anos ( $M=20,4$ anos; $D P=1,7$ anos). Foram escolhidos apenas participantes sem histórico de doença neurológica, livres de doenças oculares identificáveis e com acuidade visual normal. Todos assinaram o Termo de Consentimento Livre e Esclarecido (TCLE), conforme as normas vigentes no Brasil referente a experimentos com humanos (Resolução 196/96).

\section{Materiais e equipamentos}

O experimento foi realizado por meio de um computador com processador Intel I5 $(3,3 \mathrm{~Hz})$, monitor CRT Flatron T7305H (LG) com frequência de atualização de $85 \mathrm{~Hz}$ e teclado de resposta RB-730 (Cedrus). Os estímulos foram apresentados e as respostas gravadas pelo programa SuperLab 4.0 (Cedrus) em uma sala escura e sem som. Foi utilizado o programa Photoshop 7.0 (Adobe) para alterar o tamanho das imagens e transformá-las para escala de cinza. Um apoio para o queixo foi utilizado para posicionar adequadamente a cabeça do observador frente ao monitor, mantendo a distância de 57 $\mathrm{cm}$ entre ambos.

\section{Estímulos}

Foram utilizadas 72 fotografias do banco de imagens IAPS e 54 fotografias de faces do POFA. Em cada prova experimental, eram apresentadas duas imagens de mesma valência e modalidade. A primeira era apresentada em alta restrição temporal, chamada de "estímulo teste", e a segunda era apresentada por um tempo maior para que o participante julgasse se esta era a mesma imagem apresentada anteriormente, chamada de "estímulo comparação". Todos os estímulos foram apresentados em escala de cinza, para que o processamento de cores não afetasse a tarefa. Ademais, os estímulos teste e comparação eram apresentados em tamanhos diferentes. Ao alterarmos o tamanho das imagens formadas na retina, diminuímos a chance de que os efeitos da diferença de saliência visual entre os estímulos interferissem na tarefa de reconhecimento (seja pela imagem retida na memória de curto prazo ou por pós-efeito). Ao se considerar a dimensão prazer, as imagens do IAPS foram divididas em 24 fotografias de cenas agradáveis (imagens com filhotes de cachorro, casais se abraçando, crianças brincando e paisagens naturais, por exemplo), 24 de cenas desagradáveis (imagens de mutilados, cenas de violência com armas, cobras e acidentes naturais, por exemplo) e 24 de cenas neutras (imagens de caixa de lenços, de um ônibus, de um guarda-chuva e rodovias, por exemplo) (Tabela 1). 
Tabela 1

Listagem dos Códigos dos Estímulos Utilizados nas Provas Congruentes e Não-Congruentes (Estímulo Comparação Diferente do Estímulo Teste Entre Parênteses)

\begin{tabular}{cccc}
\hline & Agradáveis & Desagradáveis & Neutras \\
\hline 1710 & 1274 & 1390 \\
1920 & 1280 & 2575 \\
2655 & 2053 & 7550 \\
4689 & 2730 & 7640 \\
5201 & 6570 & 9210 \\
5623 & 9040 & 8116 \\
Imagens do IAPS & 9831 & 9102 & 8160 \\
& 8420 & 9220 & 9270 \\
& $1602(1603)$ & $1040(1052)$ & $7560(7595)$ \\
& $5780(5760)$ & $1200(1201)$ & $7140(7950)$ \\
& $5982(5981)$ & $6190(6200)$ & $5520(5532)$ \\
& $7450(7270)$ & $5972(5971)$ & $2485(5740)$ \\
& $8162(2791)$ & $9300(9320)$ & $1670(7150)$ \\
& $4669(4611)$ & $6350(6550)$ & $7710(7503)$ \\
$5626(7580)$ & $9410(9221)$ & $5130(7030)$ \\
& $7430(7470)$ & $3015(3063)$ & $2206(7095)$ \\
\hline
\end{tabular}

A dimensão prazer possui uma escala de 0 a 9 para cada imagem (em que 0 representa o máximo desprazer e 9 o máximo prazer). As imagens selecionadas possuem escores aproximados nas três valências existentes: agradável $(M=8,23 ; D P=0,41)$, desagradável $(M=1,85 ; D P=0,65)$ e neutra $(M=4,99 ; D P=$ $0,62)$ na normativa brasileira do IAPS (Lasaitis et al., 2008). Os estímulos comparação foram apresentados a $24 \times 18$ graus de ângulo visual $(760 \times 570$ pixels $)$ e os estímulos teste a $12 \times 9$ graus de ângulo visual $(374 \times 280$ pixels $)$. As imagens do POFA, que apresentavam equitativamente faces de homens e de mulheres, foram divididas em 15 com expressão de alegria/felicidade, 24 com expressões de medo, raiva, nojo e tristeza (divididas equitativamente) e 15 com expressão neutra (Tabela 2).

\section{Tabela 2}

Listagem dos Códigos dos Estímulos Utilizados nas Provas Congruentes e Não-Congruentes (Estímulo Comparação Diferente do Estímulo Teste Entre Parênteses)

\begin{tabular}{cccc}
\hline Agradáveis & Desagradáveis & Neutras \\
\hline A1-6 & A2-6 & MF1-2 \\
PF1-5 & C1-18 & SW3-3 \\
SW3-9 & JM2-8 & C2-3 \\
MF1-6 & MF2-7 & JM1-9 \\
WF2-12 & JJ3-12 & PE2-4 \\
& JJ4-7 & PE5-10 & GS1-4 \\
PE2-12 & WF3-16 & JB1-3 \\
Imagens do P0FA & EM4-17 & JJ3-4 \\
& JB1-9 & A1-25 (SW1-30) & C2-3 (M01-5) \\
& PF1-5 (M01-4) & JM3-11 (NR2-15) & JM1-9 (NR1-3) \\
& SW3-4 (C2-18) & C1-4 (PF2-4) & A1-2 (PF1-2) \\
MF1-6 (JM1-4) & MF1-27 (M01-23) & MF1-2 (A1-2) \\
JB1-9 (WF2-11) & PE4-5 (JB1-16) & PE2-4 (JJ3-4) \\
PE2-12 (JJ4-7) & EM5-21 (PE3-21) & JB1-3 (WF2-5) \\
JJ4-7 (EM4-7) & JB1-23 (WF3-4) & WF2-5 (EM2-4) \\
EM4-7 (GS1-8) & JJ5-5 (GS2-1) & JJ3-4 (GS1-4) \\
\hline
\end{tabular}

Os estímulos comparação foram apresentados a $16 \times 24$ graus de ângulo visual $(507 \times 760$ pixels) e os estímulos teste a $8 \times 12$ graus de ângulo visual $(259 \times 388$ pixels $)$.

\section{Procedimento e delineamento experimental}

Era explicado aos voluntários o objetivo do estudo e feita uma breve explicação acerca do experimento. Caso consentissem em participar da pesquisa, estes assinavam o TCLE e eram levados para a sala experimental. Cada participante realizava o experimento individualmente, sob as instruções do pesquisador e as descritas na telado computador.

A tarefa consistia em uma correspondência de estímulos (matchingtask). O experimento foi dividido em dois blocos diferentes, um de faces e outro de cenas. Cada bloco contava com 48 provas: 16 de cada valência (agradáveis, desagradáveis e neutras), sendo oito congruentes (estímulos teste e comparação iguais) e oito não-congruentes (estímulos teste e comparação diferentes). Os dois estímulos apresentados em cada prova não-congruente eram sempre de mesma valência. Para compor os pares de estímulos não-congruentes no bloco de cenas, procurou-se escolher imagens de semelhante complexidade visual, que não apresentavam faces frontalmente ou em grande escala para não induzir o mesmo processamento do bloco das faces e, em geral, eram de mesma temática. Para compor os pares de estímulos não-congruentes no bloco de faces, foram utilizadas imagens de faces do mesmo sexo e que expressavam a mesma emoção. O bloco de início do experimento foi contrabalançado entre os participantes. Antes do início do experimento foi realizado um treino de quatro provas, para que o participante se habituasse à tarefa.

Cada prova se iniciava com um ponto de fixação central, apresentado por $500 \mathrm{~ms}$. Logo após, o estímulo teste era apresentado durante $24 \mathrm{~ms}$ (dois frames). Uma máscara de ruído Gaussiano foi apresentada anterior e posteriormente ao estímulo subliminar (backwardeforwardmasking), durante $300 \mathrm{~ms}$ e $200 \mathrm{~ms}$, respectivamente. Esta evita a persistência retiniana (pós-efeito) do estímulo e aproxima seu tempo de processamento ao tempo de apresentação (Keysers \& Perrett, 2002). Novamente, um ponto de fixação apresentado por $500 \mathrm{~ms}$ antecedia a apresentação de outro estímulo. Este podia ser igual ou diferente ao estímulo anterior, era apresentado por 1000 ms, e somente após sua apresentação, a resposta era emitida. Por fim, era apresentada uma tela de resposta com tempo de exposição livre. A resposta do participante era dada por meio de uma de duas teclas do teclado de resposta: a) uma verde, caso o participante julgasse que o primeiro estímulo era igual ao segundo ou b) uma vermelha, caso contrário. Ao dar a resposta, era apresentada uma tela de descanso. Uma nova prova só se iniciava depois que o participante pressionasse a tecla branca central do teclado de respostas (Figura 1). 


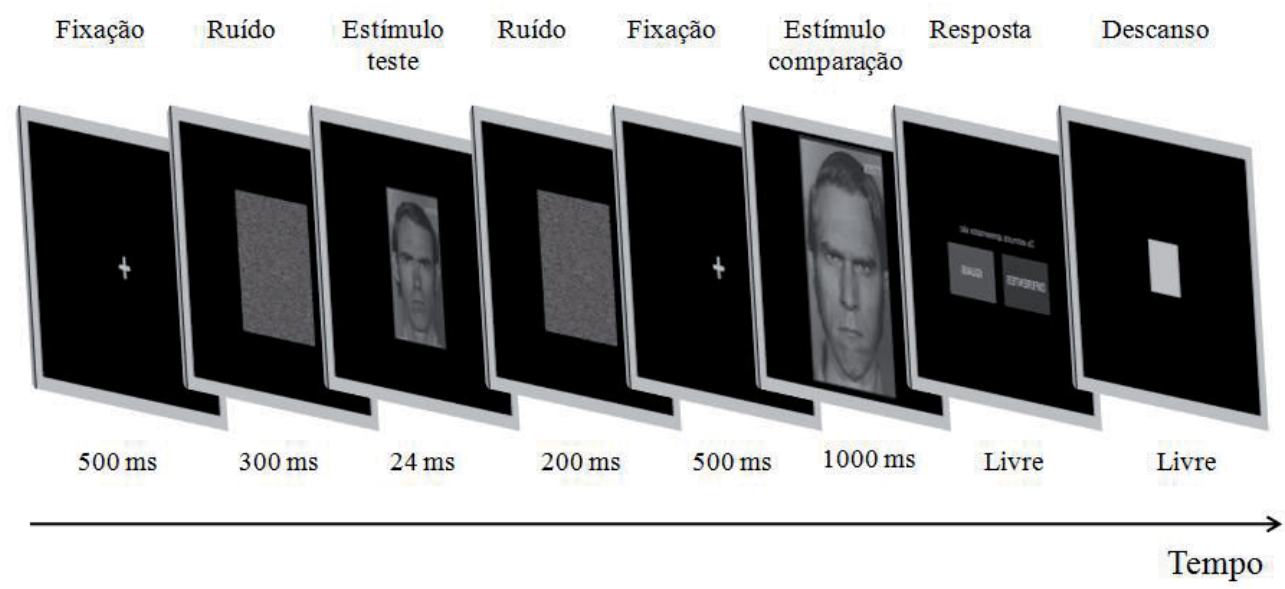

Figura 1. Esquema da Sequência de Apresentação dos Estímulos em Cada Prova Experimental

\section{Análise de dados}

Foram analisados os julgamentos e os TR (variáveis dependentes) em função da valência e modalidade dos estímulos (variáveis independentes). Para o julgamento, utilizou-se como parâmetro de desempenho a porcentagem de RC. Foram tomadas como RC os acertos e as rejeições corretas. Em relação aos TR, só foram considerados para análise aqueles tempo sem que foi emitida a resposta correta e com latência máxima de 1500 ms. As médias de RC e TR dos participantes em cada condição experimental foram submetidas a uma análise de variância de medidas repetidas [ 3 valências emocionais (neutro, agradável e desagradável) $\times 2$ modalidades de estímulo (cenas e faces)], para verificar se existiam diferenças estatisticamente significativas entre elas. Foi adotado um nível de significância de $5 \%$. A suposição de normalidade foi checada pelo teste Shapiro-Wilk. Quando foi violado o critério de esfericidade pelo teste de Mauchly, utilizou-se o ajuste de Greenhouse-Geisser. Os efeitos principais foram comparados pelo pós-teste de Bonferroni. Efeitos simples foram analisados ao se desmembrar a análise de variância para cada fator e verificar diferenças pelo mesmo pós-teste.

\section{Resultados}

\section{Análise das respostas corretas $(R C)$}

Na Figura 2 estão representadas as médias e os erros padrão das RC em cada condição experimental. Os resultados obtidos com a análise de variância apontaram efeitos principais significativos tanto da valência $\left(\mathrm{F}_{2,62}=4,34, p=0,017\right.$, $\left.\eta_{\mathrm{p}}^{2}=0,12\right)$ quanto da modalidade do estímulo $\left(\mathrm{F}_{1,31}=18,25, p<\right.$ $\left.0,001, \eta_{\mathrm{p}}^{2}=0,37\right)$. O pós-teste indicou que as $\mathrm{RC}$ foram maiores $(p<0,001)$ para estímulos neutros $(68,26)$ em relação aos desagradáveis $(62,50)$ e quando foram apresentadas cenas $(70,12)$ em comparação à faces $(61,20)$. Também foi encontrada uma intera- ção significativa entre a valência e a modalidade do estímulo $\left(\mathrm{F}_{2,52}=19,60, p<0,001, \eta_{\mathrm{p}}^{2}=0,39, \varepsilon_{\mathrm{GG}}=0,84\right)$. As demais diferenças não atingiram significância estatística. Para investigar os efeitos simples entre as condições experimentais, a análise foi desmembrada para cada estímulo e para cada valência.

Ao se analisar apenas as provas em que foram apresentadas cenas do IAPS, verificou-se uma diferença estatisticamente significativa $\left(F_{2,62}=17,13, p<0,001, \eta_{\mathrm{p}}^{2}=0,36\right)$. As médias das RC quando o estímulo visual tinha conteúdo afetivo neutro $(79,50)$ foram maiores $(p<0,001)$ do que quando apresentados estímulos de valência agradável $(67,00)$ e desagradável $(63,87)$. Ao se analisar apenas as provas em que foram apresentadas faces da série de Ekman $\left(\mathrm{F}_{2,62}=5,10, p=0,009, \eta_{\mathrm{p}}^{2}=\right.$ $0,14)$, verificou-se que as médias das $\mathrm{RC}$ quando apresentadas faces agradáveis $(65,43)$ foi maior $(p=0,012)$ em relação às neutras $(57,03)$.

Também foi realizada uma comparação entre faces e cenas para cada valência afetiva. A média de RC para provas em que foram apresentadas cenas neutras $(79,50)$ foi significativamente maior $\left(F_{1,31}=53,89, p<0,001, \eta_{p}^{2}=0,64\right)$ em relação àquelas em que foram apresentadas faces neutras $(57,03)$. A diferença de RC entre faces e cenas não foi significativa para as condições de valência emocional agradável e desagradável. Do mesmo modo, as demais diferenças de interações não atingiram significância estatística.

\section{Análise dos tempos de resposta (TR)}

Na Figura 2 estão representadas as médias e os erros padrão dos TR em cada condição experimental. Os resultados obtidos com a análise de variância não apontaram efeitos principais significativos do fator "estímulo" e da interação dos fatores "estímulo $\times$ valência" para os TR. Foi verificado efeito estatisticamente significativo apenas para a valência do estímulo $\left(\mathrm{F}_{2,62}=3,17, p=0,049, \eta_{\mathrm{p}}^{2}=0,09\right)$. O pós-teste indicou que os TR foram menores $(p=0,026)$ para estímulos agradáveis (588) em relação aos desagradáveis (640). A análise foi desmembrada 
para verificar os efeitos simples da diferença entre as valências agradável, desagradável e neutra quando apresentados cenas ou faces. Ao se analisar apenas as provas em que foram apresentadas cenas do IAPS $\left(\mathrm{F}_{2,62}=4,30, p<0,018, \eta_{\mathrm{p}}^{2}=0,12\right)$, verificou-se que as médias dos TR quando apresentadas cenas neutras (599) foi menor $(p=0,030)$ em relação às desagradáveis (673). Não foram evidenciadas diferenças significativas quando analisadas somente as provas em que foram apresentadas faces da série de Ekman. As demais diferenças não atingiram significância estatística.
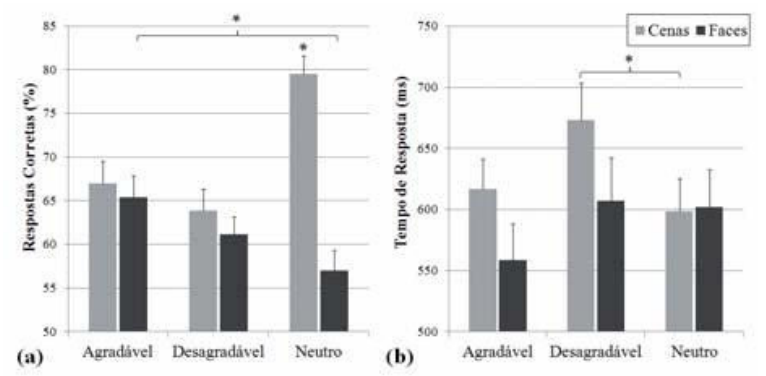

Figura 2. Médias da Porcentagem de Respostas Corretas (a) e do Tempo de Resposta (b) para Cenas de Conteúdo Emocional e Expressões Faciais de Valência Agradável, Desagradável e Neutra, Apresentadas em Alta Restrição Temporal

* diferença estatisticamente significativa $(p<0,05)$ na ANOVA. As barras verticais representam 0 erro padrão médio.

\section{Discussão}

A partir da análise dos resultados pode-se notar que, inesperadamente, cenas de valência neutra foram melhor reconhecidas que àquelas com conteúdo afetivo. Por outro lado, as faces agradáveis foram melhor reconhecidas que faces neutras. De maneira geral, a emissão de respostas para estímulos agradáveis foi mais rápida em relação aos estímulos desagradáveis.

Porém, o desempenho psicofísico alcançado pelos participantes sugere que talvez o tempo de construção das imagens na tela e sua posterior remoção podem ter ocorrido em um tempo superior ao programado, 24 ms (exposições neste tempo são consideradas subliminares). A condição de menor desempenho, faces neutras, está $7 \%$ acima da linha do acaso, 50. Apesar de calculado o número de frames e o tempo em que cada um é apresentado, o programa empregado possivelmente apresentou baixa precisão temporal quando foram utilizadas exposições muito breves. Por outro lado, delineamentos em matchingtask facilitam o posterior reconhecimento de estímulos. De qualquer modo, em novos estudos, recomenda-se que sejam utilizados programas que permitam a auditoria do real tempo de exposição e maior controle neste processo, como o Psychtoolbox, por exemplo (Kleiner, Brainard, \& Pelli, 2007).

O desempenho dos participantes no reconhecimento das cenas neutras foi superior em relação aos de valência emocional. A média desta condição foi responsável pelos efeitos principais evidenciados pela análise de variância geral realizado para as RC. O melhor reconhecimento de cenas neutras contraria a literatura que possui evidências de que estímulos visuais emocionais são mais facilmente e rapidamente percebidos em condições perceptuais limítrofes (Cuthbert et al., 2000; Hamann, 2001; Kensinger \& Schacter, 2008; Ohman et al., 2001; Vuilleumier, 2005).Porém, a prevalência no reconhecimento de flashes de cenas do IAPS de conteúdo neutro foi encontrada em estudo anterior (Komatsu, 2011). Tanto no presente trabalho como no de Komatsu (2011), as fotografias neutras foram mais facilmente reconhecidas do que as fotografias com conteúdo emocional. O resultado foi replicado mesmo com diferenças no delineamento experimental: a) uma fase de memorização antecedeu à de reconhecimento, ao invés do paradigma em matchingtask aqui adotado; b) foi utilizado o método Confidence Rating, da Teoria de Detecção de Sinal, que estima parâmetros psicofísicos a partir de curvas ROC que plotam taxas de falso alarme e acerto para cada alternativa de resposta que é dada de maneira ordinal (Fukusima \& Landeira-Fernandez, 2012); c) os estímulos eram diferentes (foram escolhidos outros estímulos do mesmo banco de imagens). Komatsu (2011) realizou uma análise qualitativa, ao comparar a complexidade e quantidade dos elementos dos estímulos neutros do IAPS utilizados, e propôs uma explicação para o efeito. Em geral, as cenas neutras são menos complexas (por exemplo, imagens de relógio, ônibus, garfo) que as de conteúdo emocional. Sendo assim, é possível que os pares de estímulos neutros julgados possuíssem maior diferença de saliência visual do que as imagens de valência emocional. O fato é agravado pela menor quantidade de estímulos neutros em relação aos classificados como agradáveis e desagradáveis do conjunto das imagens que foram normalizadas para a população brasileira, visto que é reduzida a possibilidade de escolhas, na busca de estímulos mais complexos. Esta hipótese da maior saliência dos estímulos neutros é corroborada pelos resultados do bloco de faces do POFA do experimento. As faces neutras obtiveram menores médias de $\mathrm{RC}$, seguidas pelas faces de valência negativa e positiva, em ordem crescente; sendo que as faces positivas apresentaram diferença estatística em relação às neutras. Como as faces possuem um mesmo padrão, não houve problemas com a saliência dos estímulos e, consequentemente, não houve um maior reconhecimento de estímulos menos complexos como aconteceu nas cenas neutras do IAPS. Portanto, para estudos posteriores, recomenda-se que sejam utilizadas imagens com semelhantes níveis de complexidade. Entre os controles metodológicos que podem ser adotados, citamos: a) normalizar contraste e luminância do conjunto imagens; b) utilizar um algoritmo que compare diferenças de saliência entre imagens, como os utilizados por Gutiérrez, Nummenmaa e Calvo (2009).

Em relação à modalidade dos estímulos, Britton et al. (2006) afirmaram que expressões faciais podem provocar maior reconhecimento de emoções, ao passo que as cenas induzem mais facilmente estados emotivos. Porém, nossos resultados não apontam diferenças de reconhecimento em relação à modalidade do estímulo, quando são apresentados em alta restrição temporal. Aqui, o reconhecimento de faces e 
de cenas com conteúdo emocional não apresentaram diferença significativa, apenas diferindo das neutras.

Por outro lado, o desempenho no reconhecimento das imagens foi diferente em função das valências emocionais. Na análise do TR, o reconhecimento de estímulos agradáveis foi significativemente mais rápido em relação aos estímulos desagradáveis, independente do fato de serem apresentados cenas do IAPS ou faces do POFA, quando estes foram agrupados. Ainda, cenas desagradáveis tiveram maior latência no julgamento da resposta, que se diferenciou significativamente da condição de cenas neutras. As médias das RC das faces agradáveis foi significativamente maior que a das faces neutras, que não se diferenciou das faces desagradáveis. Estes resultados sugerem um melhor processamento de estímulos agradáveis quando apresentados muito brevemente, em comparação aos estímulos desagradáveis. Este resultado também foi encontrado por Komatsu (2011), que fez apresentações subliminares de cenas do IAPS, e por D’Argembeau, Comblain e Van der Linden (2005), que fez exposições supraliminares. Bishop (2008) constatou que imagens de ameaça apresentadas rapidamente enfraquecem a ativação da amígdala e intensificam a ativação do córtex pré-frontal. A amígdala, por ser uma estrutura cerebral mais primitiva, processa as informações emocionais de uma maneira rápida, enquanto o córtex pré-frontal realiza o processamento de informações mais complexas, o que demanda maior gasto temporal. Dados a partir de indivíduos com lesão relativamente restrita à amígdala demonstraram a importância dessa região para a percepção e produção do afeto negativo e do aprendizado associativo aversivo. Essas lesões produzem uma redução geral das respostas emocionais e deficiência seletiva no reconhecimento de expressões faciais de medo (Adolphs, Tranel, Damásio, \& Damásio, 1995). Embora nem todas as imagens da condição desagradável utilizadas neste estudo tenham sido exatamente de ameaça, dada à sua natureza negativa e até mesmo aversiva, provavelmente elas foram interpretadas como ameaçadoras. É possível que as valências emocionais dos estímulos apresentados possam ter uma modalidade de processamento preferencial, realizado por estruturas cerebrais distintas. Quando apresentados em situações de exposições limítrofes, os estímulos de valência afetiva desagradável podem ser processados mais pré-frontalmente e mais lentamente do que seriam se processados pela amígdala. A ativação desta estrutura não seria atenuada pela exposição à estímulos de valência agradável, que seriam reconhecidos mais prontamente.

Cabe, por fim, ressaltar que, para investigações futuras em delineamentos semelhantes, é desejável que se aumente o número de provas por condição para que possam ser obtidas amostras de TR menos dispersas. Além disso, sugerimos que a resposta possa ser dada pelo participante na própria tela do estímulo comparação, ou que este anteceda o estímulo teste. Isto evita a possibilidade de mecanismos executivos inibitórios de uma resposta.

Em resumo, este trabalho investigou o reconhecimento de flashes de imagens de conteúdo afetivo de diferentes modalidades (faces e cenas) e valências emocionais (agradáveis, desagradáveis e neutras) por meio das RC e do TR. Em resumo, conclui-se que a simplicidade dos estímulos e a diferença de saliência perceptual entre eles podem influenciar no reconhecimento das imagens. Esta saliência pode se sobrepor aos efeitos da valência emocional dos estímulos. Ademais, o reconhecimento sob alta restrição temporal entre os estímulos de valência emocional agradável e entre os de valência desagradável são semelhantes, independente se são apresentadas faces com expressões emocionais ou cenas de conteúdo afetivo. Por outro lado, as valências apresentam diferenças: imagens agradáveis são reconhecidas mais rapidamente em relação às desagradáveis e faces agradáveis são mais reconhecidas que as neutras. Isto pode estar relacionado às estruturas cerebrais e às suas modalidades de processamento, envolvidas no comportamento emocional. O entendimento dos efeitos das valências emocionais no posterior reconhecimento de estímulos apresentados em faixas perceptuais limítrofes pode contribuir para a melhor compreensão da relação entre a emoção e o processamento cerebral. Neste estudo apontamos controles metodológicos para investigações futuras e replicamos os resultados de trabalho que antecedeu este. Os resultados são robustos frente a diferenças: do conjunto de estímulos utilizados, da amostra, de equipamentos utilizados, do delineamento experimental e estatístico.

\section{Agradecimentos}

À FAPESP, pelo apoio financeiro. Ao mestrando André Vilela Komatsu e ao professor Dr. Leonardo Gomes Bernardino, que desenvolveram estudo anterior que motivou este trabalho. Aos estudantes universitários, que consentiram em participar do experimento. Por fim, aos dois pareceristas anônimos, que contribuíram na versão final deste artigo.

\section{Referências}

Adolphs, R., Tranel, D., Damásio, H., \& Damásio, A. (1995). Fear and the human amygdala. Neuroscience, 15, 79-91.

Anderson, K., \& Phelps, E. A. (2001). Lesions of the human amygdala impair enhanced perception of emotionally salient events. Nature, 411(6835), 305-309

Bishop, S. J. (2008). Neural mechanisms underlying selective attention to threat. Annals of the New York Academy of Sciences, 1129, 141-52.

Brandão, M. L. (2004). As bases biológicas do comportamento: introdução à neurociência. São Paulo: EPU.

Britton, J. C., Taylor, S. F., Sudheimer, K. D., \& Liberzon, I. (2006). Facial expressions and complex IAPS pictures: common and differential networks. NeuroImage, 31(2), 906-919.

Bueno, J. M. H., \& Primi, R. (2003). Inteligência emocional: um estudo de validade sobre a capacidade de perceber emoções. Psicologia: Reflexão e Crítica, 16(2), 279-291.

Cuthbert, B. N., Schupp, H. T., Bradley, M. M., Birbaumer, N., \& Lang, P. J. (2000). Brain potentials in affective picture processing: covariation with autonomic arousal and affective report. Biological Psychology, 52(2), 95-111.

D’Argembeau, A., Comblain, C., \& Van der Linden, M. (2005). Affective valence and the self-reference effect: influence of retrieval conditions. British Journal of Psychology, 96, 457-466.

Damásio, A. R. (2000). O mistério da consciência. São Paulo: Companhia das Letras. 
Darwin, C. R. (2000). A expressão das emoções nos homens e nos animais ( $2^{\mathrm{a}} \mathrm{ed}$. , L. S. L. Garcia, Trad). São Paulo: Companhia das Letras (Texto original publicado em 1872).

Dixon, F. (1971). Subliminal perception: The nature of a controversy. Nova Iorque: McGraw-Hill.

Ekman, P., \& Friesen, W. V. (1976). Pictures of facial affect. Palo Alto, CA: Consulting Psychologists Press.

Ekman, P., \& Friesen, W. V. (1978). Facial action coding system: investigator's guide. Palo Alto: Consulting Psychologists Press.

Frank, M. G., \& Stennett, J. (2001). The forced-choice paradigm and the perception of facial expressions of emotion. Journal of Personality and Social Psychology, 80(1), 75-85.

Fukusima, S. S., \& Landeira-Fernandez, J. (2012). Informações complementares sobre a teoria de deteç̧ão do sinal aplicada à psicofísica. In J. Landeira Fernandez, \& S. S. Fukusima (Orgs.), Métodos em neurociência (pp. 24-33). Barueri: Manole.

Galvão, I. (2001). Expressividade e emoção: ampliando o olhar sobre as interações sociais. Revista Paulista de Educação Física, supl.4, 15-31.

Gutiérrez, A., Nummenmaa, L., \& Calvo, M. G. (2009). Enhanced processing of emotional gist in peripheral vision. The Spanish Journal of Psychology, 12(2), 414-23

Hamann, S. (2001). Cognitive and neural mechanisms of emotional memory. Trends in Cognitive Sciences, 5(9), 394-400.

Hatfield, E., Cacioppo, J. T., \& Rapson, R. L. (1992). Primitive emotional contagion. Journal of Personality and Social Psychology, 14, 151-177.

Kensinger, E. A., \& Schacter, D. L. (2008). Neural processes supporting young and older adults' emotional memories. Journal of Cognitive Neuroscience, 20(7), 1161-1173

Keysers, C., \& Perrett, D. I. (2002). Visual masking and RSVP reveal neural competition. Trends in Cognitive Sciences, 6(3), 120-125.

Kleiner, M., Brainard, D., \& Pelli, D. (2007). What's new in Psychtoolbox-3? Perception Supplement -Thirtieth European Conference on Visual Perception (p. 14). Arezzo.

Komatsu, A. V. (2011). Reconhecimento de fotografias com conteúdo emocional apresentadas subliminarmente (Monografia não publicada). Conclusão do Programa Optativo de Bacharelado em Psicologia, Universidade de São Paulo, Ribeirão Preto.

Krosnick, J. A., Metz, A. L., Jussim, L. J., \& Lynn, A. R. (1992). Subliminal conditioning of attitudes. Personality and Social Psychology Bulletin, 18, 152-162.

Lang, P. J., Bradley, M. M., \& Cuthbert, B. N. (2008). International Affective Picture System (IAPS): Affective ratings of pictures and instruction manual. Technical Report A-8, University of Florida, Gainesville, FL.

Lasaitis, C., Ribeiro, R. L., Freire, M. V., \& Amodeo, O. F. (2008). Atualização das normas brasileiras para o International Affective Picture System (IAPS). Revista de Psiquiatria do Rio Grande do Sul, 30(11), 230-235.

Mikels, J. A., Fredrickson, B. L., Larkin, G. R., Lindberg, C. M., Maglio, S. J., \& Reuter-Lorenz, P. a. (2005). Emotional category data on images from the International Affective Picture System. Behavior Research Methods, 37(4), 626-630.

Millenson, J. R. (1975). Princípios de Análise do Comportamento. Brasília: Coordenada (Obra original publicada em 1967)

Ohman, A., Lundqvist, D., \& Esteves, F. (2001). The face in the crowd revisited: a threat advantage with schematic stimuli. Journal of Personality and Social Psychology, 80(3), 381-396.

Sabatini, E., Della Penna, S., Franciotti, R., Ferretti, A., Zoccolotti, P., Rossini, P. M., Romani, G. L., et al. (2009). Brain structures activated by overt and covert emotional visual stimuli. Brain Research Bulletin, 79(5), 258-264.

Schiffman, H. R. (2001). Sensation and perception: an integrated approach (5 ed.). Nova Iorque: Wiley.

Staats, A. W. (1996). Behavior and Personality: Psychological Behaviorism. Nova Iorque: Springer Publishing Company, Inc.
Vuilleumier, P. (2005). How brains beware: neural mechanisms of emotional attention. Trends in Cognitive Sciences, 9(12), 585-594.

Whalen, P. J., Rauch, S. L., Etcoff, N. L., McInerney, S. C., Lee, M. B., \& Jenike, M. a. (1998). Masked presentations of emotional facial expressions modulate amygdala activity without explicit knowledge. The Journal of Neuroscience: the official journal of the Society for Neuroscience, 18(1), 411418.

Wild, B., Erb, M., \& Bartels, M. (2001). Are emotions contagious? Evoked emotions while viewing emotionally expressive faces: quality, quantity, time course and gender differences. Psychiatry research, 102(2), 109-24.

\section{Nota}

1. Trabalho resultante de projeto de pesquisa, requisito parcial para aprovação na disciplina de graduação Psicologia Geral e Experimental III (sobre Percepção e Psicofísica), ministrada pelo autor prof. Dr. Sérgio S. Fukusima. As autoras Beatriz F. Barbosa, Fernanda P. Garcia, Flavia H. M. Silva, Julia Ribeiro e Márcia V. Amaral formaram o grupo responsável pelo projeto. O autor Rui M. Júnior orientou o grupo ao longo do semestre letivo. Os dados deste artigo foram apresentados sob a forma de painel na $42^{\mathrm{a}}$ Reunião Anual da Sociedade Brasileira de Psicologia, em 2012. 
Rui de Moraes Júnior, Mestre em Ciências na área de Psicobiologia pela Universidade de São Paulo (USP - Ribeirão Preto), é doutorando em Ciências pelo Programa de Pós-Graduação em Psicobiologia da Universidade de São Paulo - USP, campus Ribeirão Preto e aluno visitante na École d'optometrie da Université de Montréal (UdeM). Endereço para correspondência: Rua Delmira Cândida Rodrigues da Cunha, 1093, Bairro Santa Mônica, Uberlândia-MG. CEP 38408-208. Telefone residencial no Brasil: (34) 3219-0179. Telefone celular no Canadá: (+1) 438-3452-714. E-mail: ruidemoraesjr@yahoo.com.br

Beatriz Francisco Barbosa, Graduanda em Psicologia pela Universidade de São (USP - Ribeirão Preto). E-mail: beatriz.barbosa@usp.br

Fernanda Pires Garcia, Graduanda em Psicologia pela Universidade de São Paulo (USP - Ribeirão Preto). E-mail: ferpiresg@gmail.com

Flavia Helen Moreira da Silva, Graduanda em Psicologia pela Universidade de São Paulo (USP - Ribeirão Preto). E-mail: flavia.helen.silva@gmail.com

Julia Ribeiro, Graduanda em Psicologia pela Universidade de São Paulo (USP - Ribeirão Preto). E-mail: julia2.ribeiro@gmail.com

Márcia Viana Amaral, Graduanda em Psicologia pela Universidade de São Paulo (USP - Ribeirão Preto). E-mail: marcia_amarall@hotmail.com

Sérgio Sheiji Fukusima, Doutor em Ciências na área de Psicobiologia pela Universidade de São Paulo (USP - Ribeirão Preto), é Docente no curso de Psicologia e orientador pleno no Programa de Pós-Graduação em Psicobiologia, ambos da Faculdade de Filosofia, Ciências e Letras da Universidade da São Paulo (USP Ribeirão Preto), é coordenador do Laboratório de Percepção e Psicofísica. E-mail: fukusima@usp.br 